\title{
Defect characterization in Pulsed Thermography: comparison of quantitative techniques for simulated and experimental data
}

\author{
by M. Grosso*, F. Noseda**, L.P.Caloba** and G.R.Pereira ${ }^{\star \star * *}$
}

\author{
* Laboratory of Nondestructive Testing, Corrosion and Welding, Department of Metallurgical and Materials \\ Engineering, Federal University of Rio de Janeiro, Rio de Janeiro, RJ, Brazil, marcellag@metalmat.ufrj.br \\ ${ }^{\star *}$ Applied Mathematics Department, Federal University of Rio de Janeiro, Rio de Janeiro, RJ, Brazil, \\ noseda@im.ufr.br

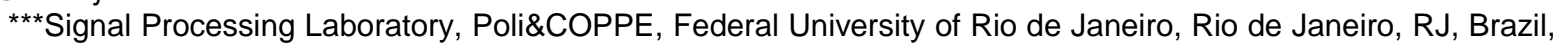 \\ caloba@|ps.ufrj.br \\ ${ }_{* * \star \star}$ Department of Metallurgical and Materials Engineering, COPPE, Federal University of Rio de Janeiro, Rio de \\ Janeiro, RJ, Brazil,gpereira@metalmat.ufrj.br
}

\begin{abstract}
A CFRP sample with inserted defects of different sizes and depths was evaluated by Pulsed Thermography. In addition to the experimental analysis, which is relative to defect detection, a computational simulation model was developed to reproduce the thermal behavior in the sample during the test. Simulations were performed with defects of several sizes and depths in order to know the influence of these parameters on the maximum thermal contrast. The performances of three models, exponential, a suitable power law and a neural network, were compared to quantitatively reproduce the data.
\end{abstract}

\section{Introduction}

Over the past decades, thermography has been considered a powerful nondestructive technique for inspection of composite materials due to many advantages, for instance, no contact with the surface, fast inspection, and easy interpretation of thermograms. Moreover, it was successfully used for the inspection of high emissivity materials, such as composite materials. However, due to the limited depth of penetration of the thermal wave, the technique is restricted to the inspection of low thickness samples [1].

The most common defects of composite materials, such as disbonds, delaminations, and impact damages can be effectively detected by active thermography. However, in addition to detection, the possibility of evaluating the size of defects by thermography would make its use more encouraging for inspection compared to other nondestructive techniques. Moreover, there are several techniques for qualitative assessment, but they are not suitable for quantitative studies such as the estimation of the size and the depth of a defect detected in a thermogram $[2,3]$. These quantitative estimations are of great practical importance since, together with fracture mechanics, they permit to assess the structural integrity of the material and the risk of keeping it in service.

The purpose of this work is to evaluate the efficiency of different techniques of data analysis in Pulsed Active Thermography. More precisely, this work aims to the quantitative estimation of depth and size of a detected defect, and we analyse both simulated and experimental data. For both simulated and experimental analysis, the sample was a CFRP (Carbon Fiber Reinforced Polymer) where several defects of different known depths and sizes were inserted.

In the first part of this work, a computational simulation model based on finite element methods was developed in order to reproduce, in three dimensions, the physical phenomena of Pulsed-Thermography inspection of this material, and data were generated in a deterministic (noise free) environment. The simulated thermal responses in defective and non defective areas during both heating and cooling were compared with experimental data in order to assess whether such a model would be valid to evaluate different configurations of defect depths and sizes. After this analysis, several simulations were performed with more defect sizes and depths in order to know the influence of these parameters on the maximum thermal contrast. With this new dataset, the performance of three different models were compared to quantitatively reproduce the data: an exponential model, a suitable power-law model and a neural-network model.

\section{Material and Methods}

\subsection{Experimental setup}

For the experimental tests, the sample was a CFRP $\left(340 \times 300 \times 4.96 \mathrm{~mm}^{3}, 16\right.$ plies $)$ contained a total of 35 Teflon $\AA$ square inclusions simulating defect of 5 lateral sizes $(3,5,7,10$ and $15 \mathrm{~mm})$ and 7 depths $(0.31,0.93,1.55,2.17$, $2.79,3.41$ and $4.03 \mathrm{~mm}$ ) as shown in figure 1. Table 1 presents the information regarding the positioning of defects in the sample. 


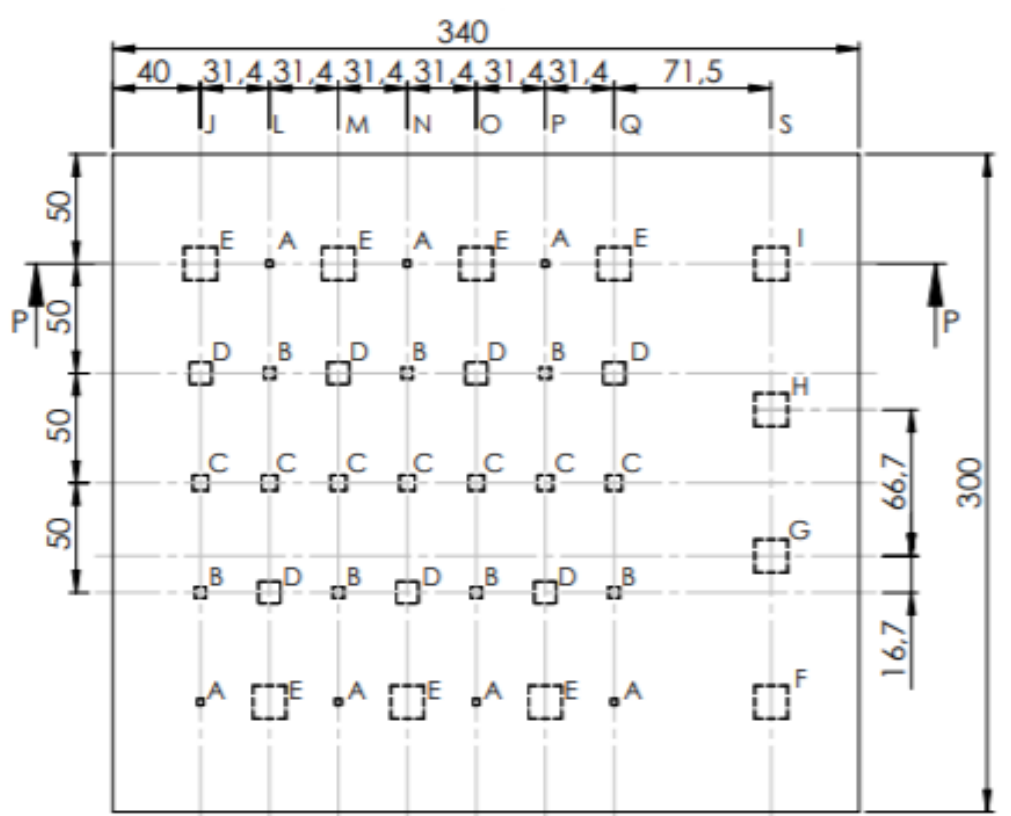

Fig. 1. Schematical drawing of CFRP sample with Teflon® inclusion defects.

Table 1. Geometric parameters of the defects inserted in the CFRP sample.

\begin{tabular}{|c|c|c|c|c|c|c|c|c|}
\hline \multirow{2}{*}{ Defect } & \multirow{2}{*}{ Size $(\mathrm{mm})$} & \multicolumn{7}{|c|}{ Depth (mm) } \\
\hline & & Row J & Row L & Row M & Row $\mathrm{N}$ & Row O & Row $\mathrm{P}$ & Row Q \\
\hline A & $3 \times 3$ & \multirow{5}{*}{0,31} & \multirow{5}{*}{0,93} & \multirow{5}{*}{1,55} & \multirow{5}{*}{2,17} & \multirow{5}{*}{2,79} & \multirow{5}{*}{3,41} & \multirow{5}{*}{4,03} \\
\hline$B$ & $5 \times 5$ & & & & & & & \\
\hline $\mathrm{C}$ & $7 \times 7$ & & & & & & & \\
\hline $\mathrm{D}$ & $10 \times 10$ & & & & & & & \\
\hline$E$ & $15 \times 15$ & & & & & & & \\
\hline
\end{tabular}

The Pulsed thermography test was carried out with an infrared camera, which is an uncooled microbolometer Focal Plane Array (FPA) camera and a set of $3,4 \mathrm{~kW}$ halogens lamps was used to generate a $10 \mathrm{~s}$ heat pulse on the material surface (reflection mode). After the heating phase, the camera collected the frames for $50 \mathrm{~s}$ of the subsequent cooling. The camera was controlled by a computer which allowed the capturing of thermal images with a frame rate of 30 $\mathrm{Hz}$.

\subsection{Computational simulation model}

To reproduce the physical phenomena that occur during the Pulsed Thermography tests, a Comsol Multiphysics $\AA$ software, version 5.1, was used to create the simulation model and the default physical model heat transfer in solid module was used. A virtual solid was created to represent the 3D geometry of the sample used in the experimental tests of this work with the corresponding inserted defects and their dimensions.

In this model, to calculate the temperature behaviour of the heat transfer principle through conduction in transient state, the Eq. 1 was used:

$$
\rho C_{p} \frac{\partial T}{\partial t}=\nabla \cdot(k \nabla T)+Q
$$

where $\rho\left(\frac{\mathrm{kg}}{\mathrm{m}^{3}}\right)$ is density, $C_{p}\left(\frac{\mathrm{J}}{\mathrm{kg} \cdot \mathrm{m}}\right)$ is the heat capacity at constant pressure, $\mathrm{T}(\mathrm{K})$ is the temperature, $\mathrm{t}(\mathrm{s})$ is the time elapsed during the test, $k\left(\frac{w}{m} \cdot K\right)$ is the material thermal conductivity and $Q\left(\frac{W}{m^{2}}\right)$ is the energy incident on the surface of the sample by the heat source. 
Two boundaries conditions were added in this model to reproduce the emission of infrared radiation issued by the surface material, Eq. 2, and the convection cooling of the surface of the material, Eq. 3, as described below:

$$
\begin{aligned}
& -n \cdot(-k \nabla T)=\varepsilon \sigma\left(T_{a m b}^{4}-T^{4}\right) \\
& -n \cdot(-k \nabla T)=h \cdot\left(T_{a m b}^{4}-T^{4}\right)
\end{aligned}
$$

where $\varepsilon$ is the emissivity, $\sigma\left(\frac{W}{m^{2} \cdot K^{4}}\right)$ is the Stefan-Boltzmann constant and $h\left(\frac{W}{m^{2} \cdot K}\right)$ is the constant convective heat transfer coefficient in the air.

In all cases, the values of each variable and physical properties contained in Eqs (1), (2) and (3) were given as parameters in the global definition of the model in the software. The mesh used was chosen according the control physics and with finer size.

\subsection{Simulations}

Several simulations were performed, with different configurations of defect depths and sizes. The size of the whole simulated sample was set to be the same as the one of the experimental sample described in Section 2.1. In the first set of simulations, the distance between the defect centers was set to be $15 \mathrm{~mm}$. Since we noticed that the thermal contrast of a defect was affected in a significant way by the presence of nearby defects (see Section 3), we changed the geometrical configuration of the defects inside the sample (Fig. 2).

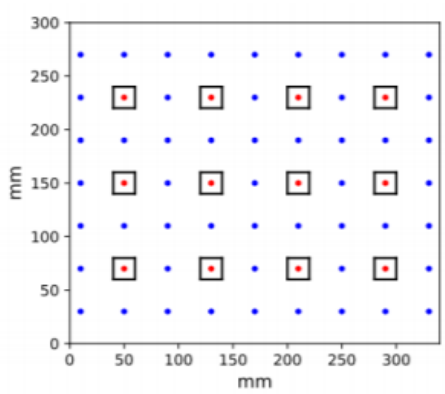

Fig. 2. Representative scheme of the defect locations in the virtual solid sample. The squares represent the defects, the blue points represent the probes in non defective regions and the red points represent the probes in the defective regions.

In the new configuration there are 12 defective regions. We made simulations for defect sizes $\mathrm{d} \times \mathrm{d} \times 0.5 \mathrm{~mm}$, where the linear size $d$ of the defect was chosen to be $(\mathrm{mm}): 5.0,7.5,10.0,12.5,15.0,17.5,20.0$. For each value of the linear defect size d, we performed 5 simulations, so that it was possible to test a total number of depths equal to $60=12 \mathrm{x}$ 5. More precisely, the tested depths $z$ were $(\mathrm{mm}): 0.06,0.12,0.18 \ldots, 3.60$. For each defect (in any of the simulations), the maximum (in time) thermal contrast $C$ was computed, where, for each instant of time $t$, the contrast was computed with respect to the background temperature, that is, the average temperature in regions without defects. In other words, the thermal contrast was computed according to the equation:

$$
T_{c}(t)=T_{d}(t)-T_{s}(t)
$$

where $T_{c}(t)$ is the absolute thermal contrast, $T_{d}(t)$ is the temperature on the defective area, and $T_{s}(t)$ is the temperature on the sound (non defective) area.

We performed three main analyses of the simulated data. More precisely, for each defect size, we made a fit of the maximum thermal contrast $\mathrm{C}(\mathrm{z})$ as function of depth for three models: exponential, a kind of power law (see below), 
and a feedforward neural network (for neural networks and machine learning in general see, for instance, [4]). The exponential model had two parameters (Eq.5):

$$
C=a_{0} e^{-a_{1} z}
$$

while the power-law model had three parameters (Eq. 6)

$$
C=\frac{a_{0}}{\left(z+a_{1}\right)^{a_{2}}}
$$

The neural network chosen was a fully-connected feedforward network with one hidden layer composed of 5 neurons. In all of the three cases the loss function to be minimized was the mean squared error. The exponential and the power model were implemented using the basic functions of TensorFlow, while for the neural network we used the TensorFlow-based library Keras.

\section{Results}

\subsection{Experimental tests}

As mentioned before, the result of a Pulsed Thermography inspection is shown as a sequence of thermographic images, in which each frame shows the temperature distribution at the surface of the material at a certain time. Fig. 3 shows the raw thermogram (without any image processing) at $11.2 \mathrm{~s}$, where most of subsurface defects can be detected.

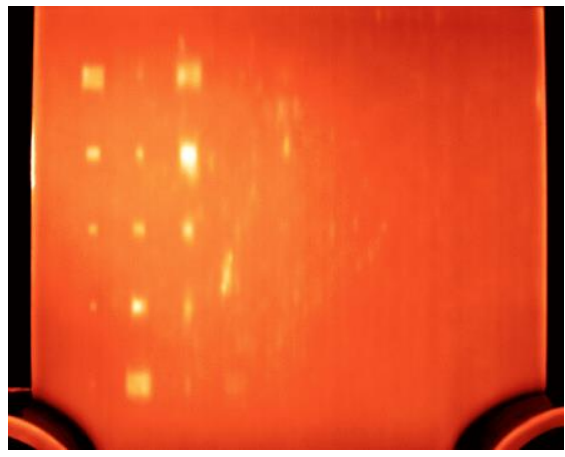

Fig. 3. Raw thermogram at 11.2 s obtained in experimental test using Pulsed Thermography.

Analysing this result, it was observed that defects positioned in row $\mathrm{J}$ (depth $=0.31 \mathrm{~mm}$ ), row $\mathrm{L}$ (depth $=0.93$ $\mathrm{mm}$ ), row $\mathrm{M}$ (depth=1.55 $\mathrm{mm}$ ) were detected in the thermogram. In row $\mathrm{N}$ (depth $=2.17 \mathrm{~mm}$ ), there is an indication of the defect of the largest size (defect $E=15 \times 15 \mathrm{~mm}$ ), however the thermal contrast presented by it is low, which does not allow us to state that is detected. Another behaviour observed from this thermogram refers to the reduction in the contrast of the defects due to reduction of their size and the increase in their depth, as predicted in the literature.

\subsection{Validation of the computation simulation model created}

As in the experimental tests, the result obtained from this computational simulation model is presented in the form of images illustrating the thermal distribution on the surface of the material as a function of time and also curves of 
temperature evolution over time for material regions selected by the user. Fig. 4 shows the image with the temperature distribution on the sample surface obtained by the computational simulation model proposed in this study.

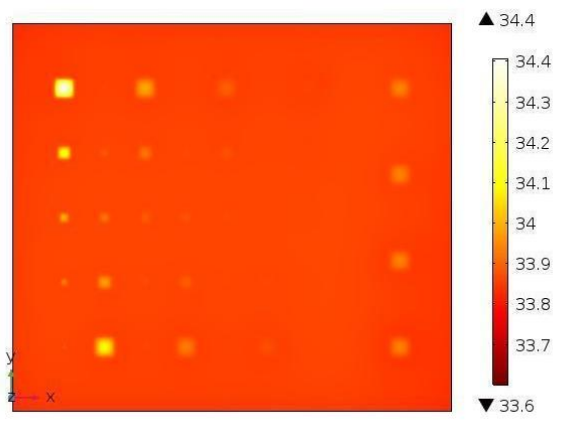

Fig. 4. Simulated thermogram at 11 s obtained.

Analysing the resulting images obtained by the experimental tests (Fig. 3) with the image resulting from the computational simulation model proposed in this study (Fig. 4), a similar behaviour regarding the detection of defects related to the depth of the themselves. However, in addition to the visual analysis, it was decided to evaluate the efficiency of this model as a tool capable of virtually reproducing the thermal behaviour during the inspection of this sample by another criterion: comparison of the results of temperature evolution for regions of defects and without defects through the two methodologies. The temperature curves for each defect detected comparing the results obtained for both methodologies are shown in Fig.5, Fig.6 and Fig. 7. A similar comparison is shown in Fig. 8 relative to the regions without defects.

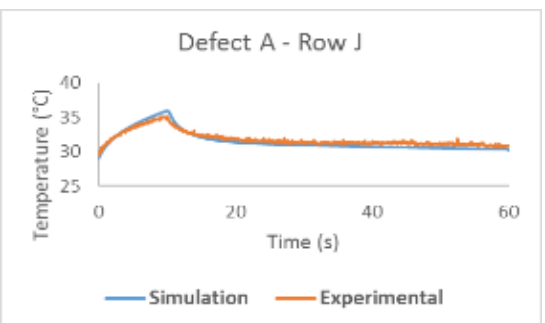

(a)

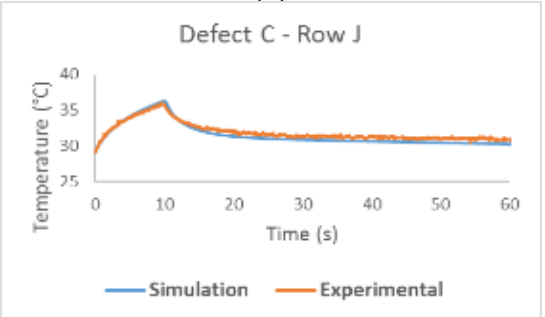

(c)

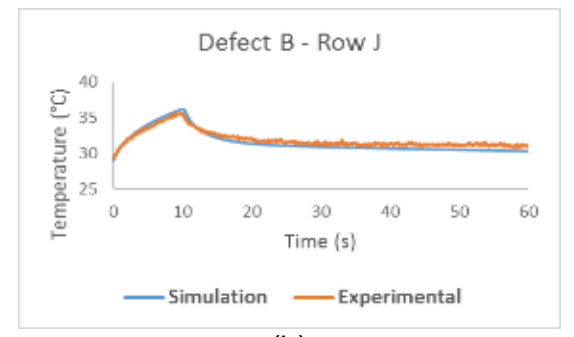

(b)

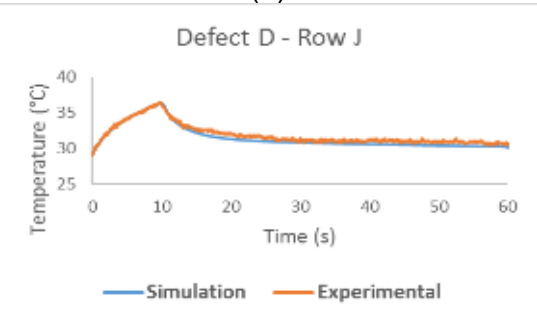

(d)

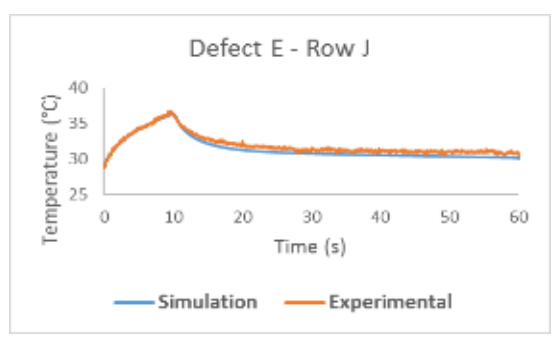

(e)

Fig. 5. Temperatures curves obtained by simulation and experimental tests in row $J$ (depth $=0.31 \mathrm{~mm}$ ) for defect: (a) $A$ (size = $3 \times 3 \mathrm{~mm}),($ b) $B($ size $=5 \times 5 \mathrm{~mm}),(c) C($ size $=7 \times 7 \mathrm{~mm})$, (d) $D($ size $=10 \times 10 \mathrm{~mm})$ and $(e) E($ size $=15 \times 15 \mathrm{~mm})$. 


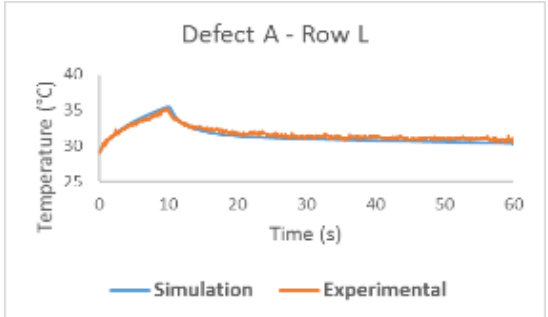

(a)

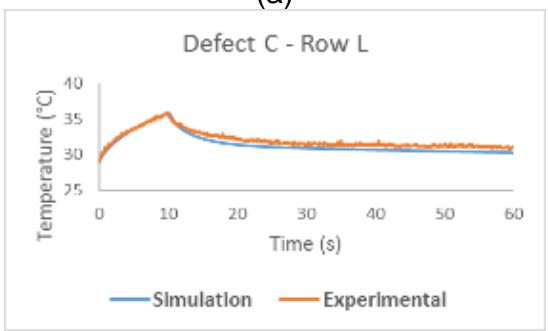

(c)

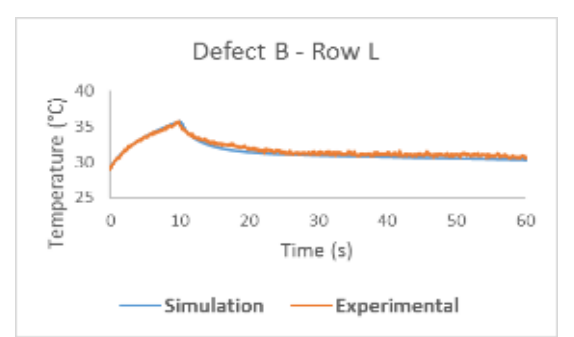

(b)

Defect D - Row L

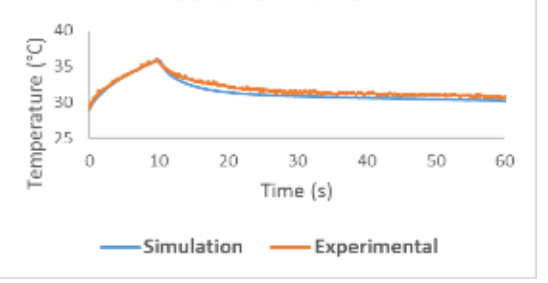

(d)

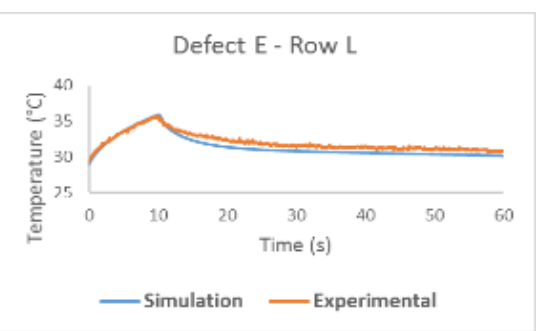

(e)

Fig. 6. Temperatures curves obtained by simulation and experimental tests in row $L$ (depth $=0.31 \mathrm{~mm}$ ) for defect: (a) $A($ size $=3 \times 3 \mathrm{~mm})$, (b) $B($ size $=5 \times 5 \mathrm{~mm}),(c) C($ size $=7 \times 7 \mathrm{~mm})$, (d) $D($ size $=10 \times 10 \mathrm{~mm})$ and $(e) E($ size $=15 \times 15 \mathrm{~mm})$.

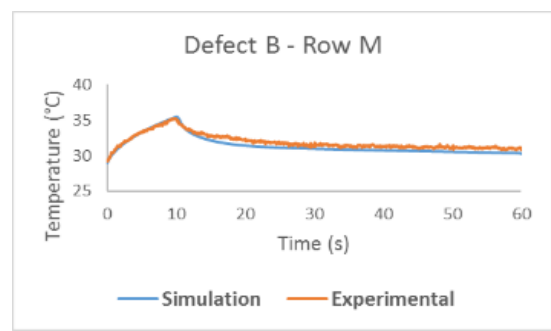

(a)

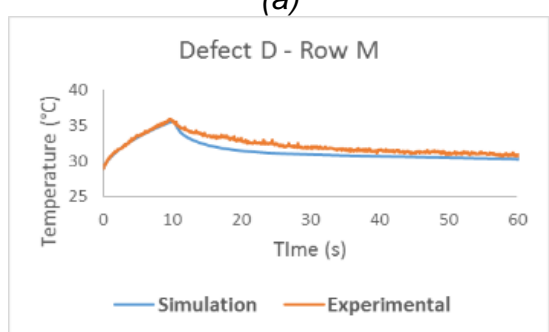

(c)

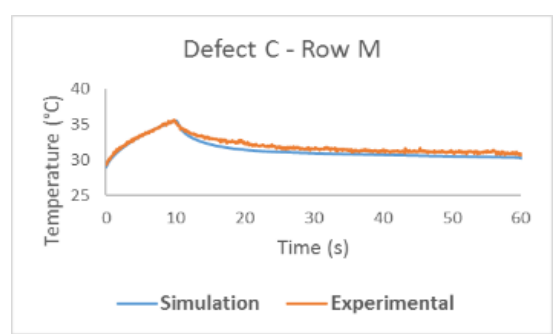

(b)

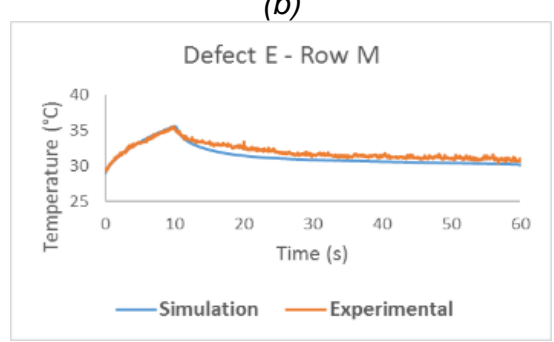

(d)

Fig. 7. Temperatures curves obtained by simulation and experimental tests in row $M$ (depth $=0.31$ $\mathrm{mm}$ ) for defect: (a) $B($ size $=5 \times 5 \mathrm{~mm})$, (b) $C($ size $=7 \times 7 \mathrm{~mm}),(\mathrm{c}) D($ size $=10 \times 10 \mathrm{~mm})$ and (d) $E$ (size $=15 \times 15 \mathrm{~mm})$. 


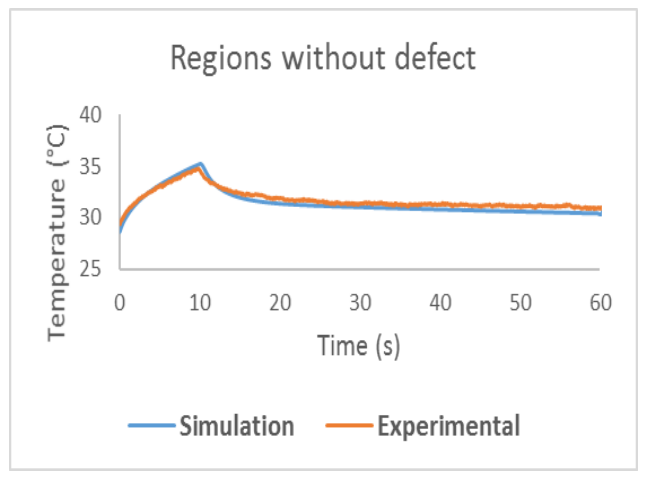

Fig. 8. Temperatures curves obtained by simulation and experimental tests for regions without defect.

According to the results presented above, it was found that the computational simulation model developed in this study could be validated due to its great similarity with the experimental data.

\subsection{Simulation results and quantitative analysis}

In Fig. 9 it is shown the maximum thermal contrast as a function of depth for defect size $15 \mathrm{~mm} \times 15 \mathrm{~mm} \times 0.5$ $\mathrm{mm}$. These data are relative to a simulation with nearby defects as explained in Section 2.3. The defects were placed in a rectangular grid with depth increasing from left to right and from bottom to top. The curve shows some "bumps" and inflection points, and each bump has a number of points equal to the number of defects present in a row. This behaviour shows that the thermal contrast was influenced by the "geometry", which seems to indicate that the defects had a thermal interaction due to their proximity, as expected on an intuitive ground. The curves for the other defect sizes presented the same behaviour.

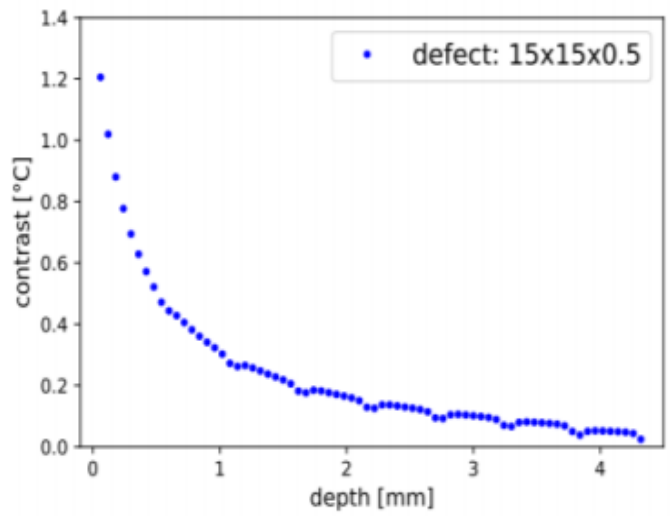

Fig. 9. The thermal-contrast curve for nearby defects exhibits some inflexions points.

According to the final chosen geometry (Fig.2), Fig. 10 shows the data relative to all the seven defect sizes. We observe that the bumps mentioned above are absent, which confirms both the reliability of the new data and the hypothesis that nearby defects "interact". In the sequel, for clarity, we present the results relative to selected defect sizes; for the other sizes the behaviour is similar. Fig. 11 shows the fit of the exponential model for one defect size. Fig. 12 shows the fit of the power-law model for two different defect sizes. Fig. 13 shows the value of the parameters for this model for defect size $d$ $x d \times 0.5 \mathrm{~mm}$, for all the simulated values of d. Finally, Fig. 14 shows the best fit for the neural-network model. 


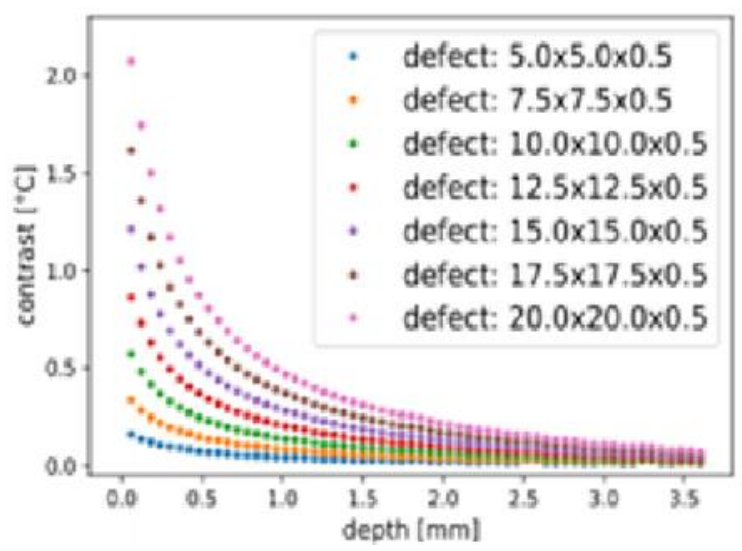

Fig. 10. Thermal contrast as function of depth for different defect sizes (simulated data).

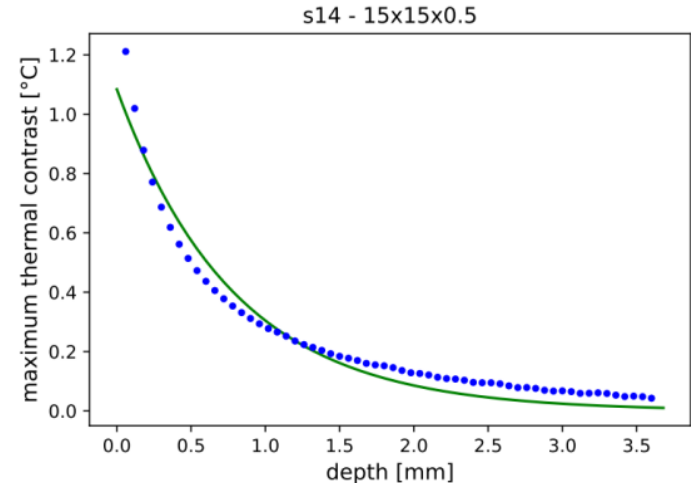

Fig.11. The blue points are simulated data and the green curves is the best fit of the exponential model according to least squares method.

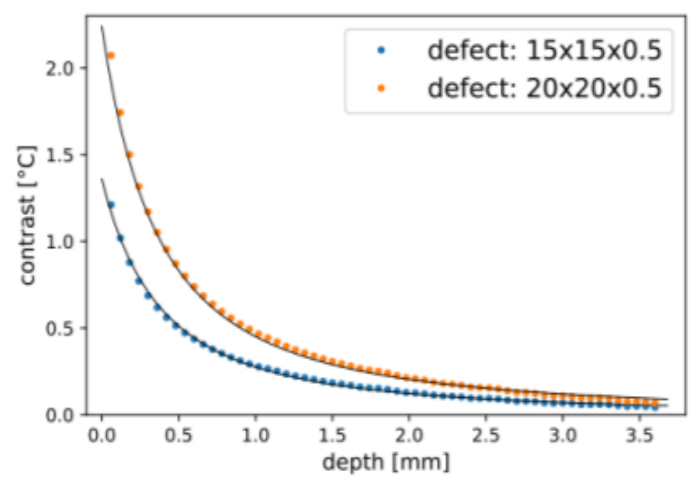

Fig. 12. Thermal contrast for simulated data (blue and orange points) and power-model fit (black curve) for two different defect sizes. 


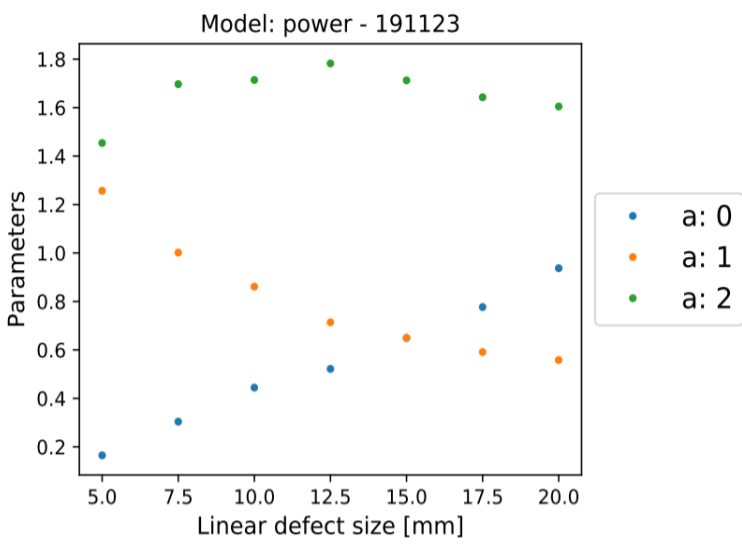

Fig. 13. The plot shows the values of the parameters ao (blue), a1 (orange) and a2 (green) of the power-law model for different values of the linear size $d$.

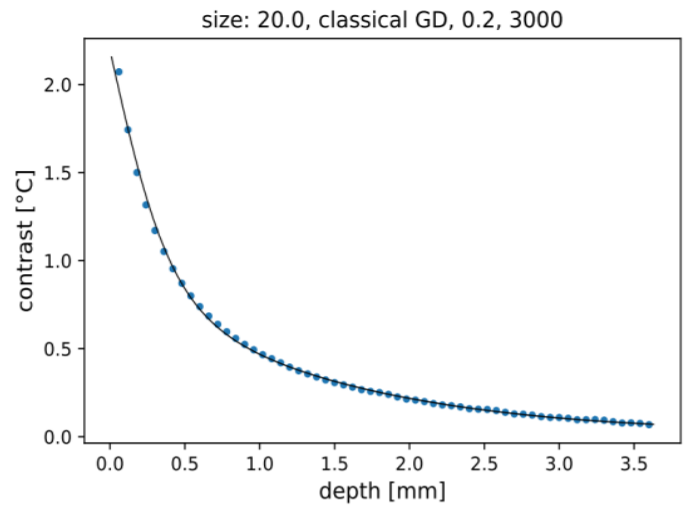

Fig. 14. The points represent the simulated data while the continuous curve is the best fit for the neural-network model according to least squares method.

\section{Conclusions}

According to the experimental tests, in this work the pulsed modality was able to detect all defects inserted in the sample to a depth of $1.55 \mathrm{~mm}$. Such behaviour indicates that the detection limitation presented by this modality is between $1.55 \mathrm{~mm}$ and $2.17 \mathrm{~mm}$ (in this depth, only the biggest defect was identified). With these results, the continuation of this study is encouraged to assess post-processing techniques and also other types of thermography that can corroborate to define the detection limit. Regarding the computational simulation model developed in this study to reproduce the thermal behaviour in this material during the Pulsed Thermography tests, the results obtained showed a great similarity with the experimental data, so that it was validated and became an extremely useful tool for generating simulated data for the quantitative analysis of defects.

Concerning the simulated data and the fit of the thermal contrast $\mathrm{C}(\mathrm{z})$ as a function of depth for fixed defect size, the exponential model presents a systematic error that makes it unsatisfactory. On the other hand, the proposed powerlaw model looks quite interesting since, with only three parameters, it permits to satisfactorily describe the data. As a proposal for future work, one may further investigate the behaviour of the parameters of the power-law model (for instance, for different materials); due to the simplicity of the model, one may try to find a physical explanation of the law in theoretical terms. As far as the neural-network mode is concerned, it fits the data as satisfactorily as the power-law model. We observe that, for future improvements and applications of these results, the use of neural networks is preferable for the following reason. The models we tested are function of just one variable (depth) and it seems not obvious to find a simple explicit analytic expression (like the proposed power-law model) for the contrast in terms of depth and size, which would be desirable. Using neural networks, one gives up on simplicity but gains a working method to approach the problem. This direction will be pursued in future works. 


\section{REFERENCES}

[1] Maldague, X.P.V., 'Infrared and Thermal Testing - non destructive testing', ASNT; 2001.

[2] Peeters J., Ibarra-Castanedo C., Sfarra S., et al., Robust quantitative depth estimation on CFRP samples using active thermography inspection and numerical simulation updating, NDT\&E International. - Vol 87, pp. 119-123, 2017.

[3] Saeed N., Abdulrahman Y., Amer S., Omar M., Experimentally validated defect depth estimation using artificial neural network in pulsed thermography. - Vol 98, pp. 192-200, 2019.

[4] C. M. Bishop, "Pattern recognition and machine learning", Springer, 2006. 